\title{
Equivalence classes of exponential polynomials with the same set of zeros
}

\author{
J.M. Sepulcre* and T. Vidal \\ Department of Mathematical Analysis, University of Alicante, 03080-Alicante, Spain.
}

(May 2014)

\begin{abstract}
Through several equivalence binary relations, in this paper we identify, on the one hand, groups of exponential polynomials with the same set of zeros, and on the other, groups of functional equations of the form $a_{1} f\left(\gamma_{1} z\right)+a_{2} f\left(\gamma_{2} z\right)+\ldots+a_{n} f\left(\gamma_{n} z\right)=$ $0, z \in \mathbb{C}$ that lead to equivalent exponential polynomials with the same set of zeros.
\end{abstract}

Keywords: Zeros of Entire Functions; Exponential Polynomials; Functional equations.

AMS Subject Classification: 30Axx; 30D05, 39Bxx.

\section{Introduction}

For each integer $n \geq 1$, let

$$
P(z):=a_{1} e^{\alpha_{1} z}+\ldots+a_{n} e^{\alpha_{n} z}, z \in \mathbb{C},
$$

be an exponential polynomial of non-null complex coefficients $a_{1}, \ldots, a_{n}$ and distinct frequencies $\alpha_{1}, \ldots, \alpha_{n}$ so arranged that $\alpha_{i}$ comes before $\alpha_{j}$ if $\operatorname{Re}\left(\alpha_{i}\right)<\operatorname{Re}\left(\alpha_{j}\right)$ or if $\operatorname{Re}\left(\alpha_{i}\right)=\operatorname{Re}\left(\alpha_{j}\right)$ but $\operatorname{Im}\left(\alpha_{i}\right)<\operatorname{Im}\left(\alpha_{j}\right)$. The study of the zeros of the exponential polynomials $P(z)$ is a topic which appears in the first third of the twentieth century in relation with the development of differential equation theory (see for example [9]). Let $C_{P}$ denote the convex polygon in the complex plane defined by the complex conjugates of the frequencies of $P(z)$, that is, the convex hull of the points $\overline{\alpha_{1}}, \ldots, \overline{\alpha_{n}}$, then the zeros of $P(z)$ lie in half-strips in the directions of the exterior normals to $C_{P}$. In the extensive literature about this topic, we can find for example several formulae to determine the number of its zeros in an arbitrary region of each one of the half-strips where they are situated (see for example [4], [7] and [8]).

The first purpose in this paper is to identify groups of exponential polynomials $P(z)$ which have the same set of zeros and to characterize them through some equivalence binary relations. To do it, we will consider generic exponential polynomials in Section 2 and, subsequently, we will handle particular classes of these polynomials with real and, more generally, aligned frequencies in sections 3 and 4 respectively. In this manner, the exponential polynomials with the same set of zeros will be able to be directly identified from their respective classes.

\footnotetext{
*Corresponding author. Email: JM.Sepulcre@ua.es
} 
On the other hand, the exponential polynomials $P(z)$ are closely related to the functional equations of the form

$$
a_{1} f\left(\gamma_{1} z\right)+a_{2} f\left(\gamma_{2} z\right)+\ldots+a_{n} f\left(\gamma_{n} z\right)=0, z \in \mathbb{C},
$$

where the $a_{j}$ 's and the $\gamma_{j}$ 's are non-null complex numbers and $n \geq 2$ is an integer number. In fact, we will see that each functional equation of the form above has associated an exponential polynomial $a_{1} e^{z \log \gamma_{1}}+a_{2} e^{z \log \gamma_{2}}+\ldots+a_{n} e^{z \log \gamma_{n}}$ of type (1.1), where $\log z$ denotes the principal branch of the logarithm. It is worth noting that this property was already shown for the special cases

$$
f(z)+f(2 z)+\ldots+f(n z)=0, n \in \mathbb{N}, n \geq 2,
$$

introduced in the literature by Mora, Cherruault and Ziadi in 1999 [3], and used for modeling certain processes related to combustion of hydrogen in a car engine for small values of $n[5]$.

Thus, concurrently with the development of the different equivalence classes of exponential polynomials, the second purpose in this paper is to establish equivalence binary relations on different classes of functional equations of the form (1.2) that lead us to corresponding equivalent exponential polynomials with the same set of zeros. Furthermore, we will prove that the converse is true in the sense that two equivalent exponential polynomials also lead us to two equivalent functional equations. As in the case of the exponential polynomials, we will establish more specific equivalence classes of the functional equations above which preserve the same properties as in the general case.

Interestingly, it was proved in $[5]$ that every zero of the exponential polynomial $1+2^{z}+\ldots+n^{z}, n \in \mathbb{N}, n \geq 2$, provides a vector space of basic solutions of functional equation (1.3). Furthermore, for the case $n=2$ other solutions appear by considering the pe function of Weierstrass [5, Section 4] or the binary characteristic of a real number [6, Proposition 2.3]. Also, the solutions not identically null on the rationals of Cauchy functional equation and the ternary and quaternary characteristics of a real number provide solutions for the cases $n=3$ and $n=4$ respectively [6, Theorem 2.7, Theorem 2.11]. Furthermore, in a recent paper [1], the authors consider the equation

$$
f(z)+f\left(a_{1} z\right)+\ldots+f\left(a_{N} z\right)=0,
$$

where $0<a_{1}<a_{2}<\ldots<a_{N}$ are positive real numbers and $N \in \mathbb{N}$ with $N \geq 2$, and they study the existence of continuous periodic solutions. As a consequence of the above, there exist functional equations of the form (1.2) which have solutions that apparently are not linked to their corresponding polynomials. In spite of this, the equivalence binary relations that we will establish on the functional equations above, that will lead us to corresponding equivalent exponential polynomials, determine equivalent functional equations that exactly have the same set of solutions defined on $\mathbb{C}$, not uniquely the basic solutions which arise from the corresponding exponential polynomials.

\section{Generic complex frequencies}

We start by considering generic exponential polynomials of complex coefficients and frequencies. 
Definition 2.1 An exponential polynomial is said to be of the class $\mathcal{P}$ if it is of the form (1.1).

Note that the set $\mathcal{P}$ of functions of the form (1.1) is closed with respect to the sum and the multiplication; that is, the sum or the product of any two functions of the class is also in the class. Furthermore, the derivative of an element of the class is also in the class.

We can also consider the following subclass of exponential polynomials with only one term.

Definition 2.2 An exponential polynomial is said to be of the class $\mathcal{T} \subset \mathcal{P}$ if it is of the form $c e^{\lambda z}$ with $c, \lambda \in \mathbb{C}$.

It is immediate that the class $\mathcal{T}$ is also closed with respect to the sum and multiplication, the derivative of any element of $\mathcal{T}$ is in the same class and each non-null element $T(z)=c e^{\lambda z}$ in $\mathcal{T}$ has an inverse element $T^{-1}(z)=\frac{1}{c} e^{-\lambda z}$ in the class.

We next identify two elements in the class $\mathcal{P}$ in terms of the class $\mathcal{T}$.

Definition 2.3 We will say that two elements $P(z), Q(z) \in \mathcal{P}$ are equivalent, and it will be denoted by $P(z) \sim Q(z)$, when there exists $T(z) \in \mathcal{T}$ such that $P(z)=T(z) \cdot Q(z)$ for all $z \in \mathbb{C}$.

Notice that the binary relation $\sim$ defined above on the class $\mathcal{P}$ is an equivalence relation. Indeed, if $P(z), Q(z)$ and $R(z)$ are non-null elements of $\mathcal{P}$, we have that

i) (Reflexivity) As $1 \in \mathcal{T}$ then $P(z) \sim P(z)$;

ii) (Symmetry) If $P(z) \sim Q(z)$ then there exists a non-null $T(z) \in \mathcal{T}$ such that $P(z)=T(z) \cdot Q(z)$. Hence, since $Q(z)=T^{-1}(z) \cdot P(z)$ and $T^{-1}(z) \in \mathcal{T}$, then $Q(z) \sim P(z)$

iii) (Transitivity) If $P(z) \sim Q(z)$ and $Q(z) \sim R(z)$ then there exist $T_{1}(z)$ and $T_{2}(z) \in \mathcal{T}$ such that $P(z)=T_{1}(z) \cdot Q(z)$ and $Q(z)=T_{2}(z) \cdot S(z)$. Hence $P(z)=T(z) \cdot R(z)$ with $T(z):=T_{1}(z) \cdot T_{2}(z) \in \mathcal{T}$ and, consequently, $R(z) \sim$ $P(z)$.

It is an elementary check that two equivalent exponential polynomials have the same number of distinct frequencies.

Given $P(z) \in \mathcal{P}$, let $Z(P)$ denote the set $\{z \in \mathbb{C}: P(z)=0\}$ of the zeros of $P(z)$. With the help of Hadamard's representation it is easy to characterize the equivalent exponential polynomials depending on their zeros.

Proposition 2.4 Let $P(z)$ and $Q(z)$ be two exponential polynomials in the class $\mathcal{P}$. Thus $P(z) \sim Q(z)$ if and only if $Z(P)=Z(Q)$ and their zeros have associated the same multiplicity.

Proof. Let $P(z)$ and $Q(z)$ be two exponential polynomials in the same equivalence class of $\mathcal{P} / \sim$, then there exists $T(z)=c e^{\lambda z} \in \mathcal{T}$ such that

$$
P(z)=T(z) Q(z)
$$

It is immediate that if $Z(Q)=\emptyset$ then $Z(P)=\emptyset$, thus let $z_{0} \in Z(Q)$ be a zero of $Q(z)$ of multiplicity $k \geq 1$. Hence,

$$
Q(z)=\left(z-z_{0}\right)^{k} g(z)
$$


where $g(z)$ is an entire function such that $g\left(z_{0}\right) \neq 0$ and

$$
Q\left(z_{0}\right)=Q^{\prime}\left(z_{0}\right)=Q^{\prime \prime}\left(z_{0}\right)=\ldots=Q^{(k-1)}\left(z_{0}\right)=0, Q^{(k)}\left(z_{0}\right) \neq 0 .
$$

Now, since $P(z)=c e^{\lambda z} g(z)\left(z-z_{0}\right)^{k}$, then $P\left(z_{0}\right)=0$. Furthermore, since $P(z)=$ $c e^{\lambda z} Q(z)$, then

$$
P^{\prime}\left(z_{0}\right)=C e^{\lambda z_{0}}\left(\lambda Q\left(z_{0}\right)+Q^{\prime}\left(z_{0}\right)\right)
$$

and, more generally, we have

$$
P^{(m)}\left(z_{0}\right)=C e^{\lambda z_{0}} \sum_{j=0}^{m}\left(\begin{array}{c}
m \\
j
\end{array}\right) \lambda^{m-j} Q^{(j)}\left(z_{0}\right), 1 \leq m \leq k .
$$

Therefore, it is also verified that

$$
P\left(z_{0}\right)=P^{\prime}\left(z_{0}\right)=P^{\prime \prime}\left(z_{0}\right)=\ldots=P^{(k-1)}\left(z_{0}\right)=0, P^{(k)}\left(z_{0}\right) \neq 0
$$

and $z_{0}$ is a zero of $P(z)$ of multiplicity $k$.

Conversely, suppose now that $P(z), Q(z) \in \mathcal{P}$ are so that

$$
Z(P)=Z(Q)=\left\{z_{1}, z_{2}, \ldots, z_{l}, \ldots\right\}
$$

and their zeros have associated the same multiplicity. Then their quotient $\frac{P(z)}{Q(z)}$ is an entire function of exponential type without zeros. Hence, by Hadamard factorization theorem [2, Theorem 4.4.3], it is an exponential monomial or, equivalently,

$$
P(z)=c e^{\lambda z} Q(z)
$$

for some $c, \lambda \in \mathbb{C}, c \neq 0$. Consequently $P(z) \sim Q(z)$.

On the other hand, we will introduce a binary relation on the class of functional equations of the form (1.2), that is

$$
a_{1} f\left(\gamma_{1} z\right)+a_{2} f\left(\gamma_{2} z\right)+\ldots+a_{n} f\left(\gamma_{n} z\right)=0, z \in \mathbb{C},
$$

where the $a_{j}$ 's and also the $\gamma_{j}$ 's are non-null complex numbers and $n \geq 2$ is an integer number.

Before going to this, it is worth noting that each functional equation of the form (1.2) has associated the exponential polynomial $P_{n}(z)=a_{1} e^{z \log \gamma_{1}}+a_{2} e^{z \log \gamma_{2}}+$ $\ldots+a_{n} e^{z \log \gamma_{n}}$ which belongs to the class $\mathcal{P}$, where $\log z$ is the principal branch of the logarithm. Indeed, if $f_{n, j}(z)=e^{\beta_{n, j} \log z}$, where $\beta_{n, j}$ is a zero of $P_{n}(z)$, then

$$
\begin{gathered}
a_{1} f_{n, j}\left(\gamma_{1} z\right)+a_{2} f_{n, j}\left(\gamma_{2} z\right)+\ldots+a_{n} f\left(\gamma_{n} z\right)=0 \\
a_{1}\left(\gamma_{1} z\right)^{\beta_{n, j}}+a_{2}\left(\gamma_{2} z\right)^{\beta_{n, j}}+\ldots+a_{n}\left(\gamma_{n} z\right)^{\beta_{n, j}}= \\
z^{\beta_{n, j}}\left(a_{1} \gamma_{1}^{\beta_{n, j}}+a_{2} \gamma_{2}^{\beta_{n, j}}+\ldots+a_{n} \gamma_{n}^{\beta_{n, j}}\right)=
\end{gathered}
$$




$$
z^{\beta_{n, j}} P_{n}\left(\beta_{n, j}\right)=0
$$

and, hence, the functions

$$
g_{n, j}(z)=\left\{\begin{array}{ll}
f_{n, j}(z) & \text { if } z \neq 0 \\
0 & \text { if } z=0
\end{array},\right.
$$

which are closely related to the exponential polynomials $P_{n}(z)$, provide solutions defined on $\mathbb{C}$ to the functional equation above.

Definition 2.5 Let

$$
a_{1} f\left(\gamma_{1} z\right)+a_{2} f\left(\gamma_{2} z\right)+\ldots+a_{n} f\left(\gamma_{n} z\right)=0, z \in \mathbb{C}
$$

be a functional equation of the form (1.2), then

$$
P_{n}(z)=a_{1} e^{z \log \gamma_{1}}+a_{2} e^{z \log \gamma_{2}}+\ldots+a_{n} e^{z \log \gamma_{n}}
$$

will be called the corresponding exponential polynomial.

Definition 2.6 For each integer number $n \geq 2$, let $\mathcal{F}_{n}$ be the set of all functional equations of the form (1.2) with $n$ terms. We will say that two functionals equations in $\mathcal{F}_{n}$,

$$
a_{1} f\left(\gamma_{1} z\right)+a_{2} f\left(\gamma_{2} z\right)+\ldots+a_{n} f\left(\gamma_{n} z\right)=0, z \in \mathbb{C}
$$

and

$$
b_{1} f\left(\delta_{1} z\right)+b_{2} f\left(\delta_{2} z\right)+\ldots+b_{n} f\left(\delta_{n} z\right)=0, z \in \mathbb{C}
$$

are equivalent when there exist $\lambda \in \mathbb{C}$ and $c \in \mathbb{C} \backslash\{0\}$ such that $\delta_{j}=e^{\lambda} \gamma_{j}$ and $b_{j}=c a_{j}$ for each $j=1,2, \ldots, n$.

Plainly, the binary relation defined above on the class $\mathcal{F}_{n}$ is an equivalence relation.

We next prove that two exponential polynomials which are associated to two equivalent functional equations have the same set of zeros.

Proposition 2.7 For each $n \geq 2$, let $P_{n}(z)$ and $Q_{n}(z)$ be the corresponding exponential polynomials associated to two equivalent functional equations in $\mathcal{F}_{n}$, then $P_{n}(z) \sim Q_{n}(z)$.

Proof. Let

$$
a_{1} f\left(\gamma_{1} z\right)+a_{2} f\left(\gamma_{2} z\right)+\ldots+a_{n} f\left(\gamma_{n} z\right)=0, z \in \mathbb{C}
$$

and

$$
b_{1} f\left(\delta_{1} z\right)+b_{2} f\left(\delta_{2} z\right)+\ldots+b_{n} f\left(\delta_{n} z\right)=0, z \in \mathbb{C}
$$

be two equivalent functional equations in $\mathcal{F}_{n}$ and $c \in \mathbb{C} \backslash\{0\}, \lambda \in \mathbb{C}$ such that $\delta_{j}=e^{\lambda} \gamma_{j}$ and $b_{j}=c a_{j}$ for each $j=1,2, \ldots, n$. Their associated exponential 
polynomials are

$$
P_{n}(z)=a_{1} e^{z \log \gamma_{1}}+a_{2} e^{z \log \gamma_{2}}+\ldots+a_{n} e^{z \log \gamma_{n}}
$$

and

$$
Q_{n}(z)=b_{1} e^{z \log \delta_{1}}+b_{2} e^{z \log \delta_{2}}+\ldots+b_{n} e^{z \log \delta_{n}}
$$

respectively. Now, check that $Q_{n}(z)=c e^{\lambda z} P_{n}(z)$ and, since $c e^{\lambda z}$ has no zeros, then the sets $Z\left(P_{n}\right)$ and $Z\left(Q_{n}\right)$ are the same. Finally, from Proposition 2.4, we have $P_{n}(z) \sim Q_{n}(z)$.

We are now ready to prove that the converse of Proposition 2.7 is also true.

Proposition 2.8 Let $P(z)$ and $Q(z)$ be two exponential polynomials in $\mathcal{P} \backslash \mathcal{T}$ such that $P(z) \sim Q(z)$. Thus $P(z)$ and $Q(z)$ are the corresponding exponential polynomials associated to two equivalent functional equations in $\mathcal{F}_{n}$ for some integer number $n \geq 2$.

Proof. As $P(z) \sim Q(z)$, then $P(z)=T(z) Q(z)$ with $T(z)=c e^{\lambda z}, c \in \mathbb{C} \backslash\{0\}$ and $\lambda \in \mathbb{C}$. Therefore, if $P(z) \in \mathcal{P}$ is of the form

$$
P(z)=a_{1} e^{\alpha_{1} z}+\ldots+a_{n} e^{\alpha_{n} z}
$$

for some $n \geq 2$ then

$$
Q(z)=b_{1} e^{\beta_{1} z}+\ldots+b_{n} e^{\beta_{n} z}
$$

with $b_{j}=c a_{j}$ and $\beta_{j}=\alpha_{j}+\lambda$ for $j=1,2, \ldots, n$.

Thus, $P(z)$ is the corresponding exponential polynomial associated to the functional equation

$$
a_{1} f\left(e^{\alpha_{1}^{*}} z\right)+a_{2} f\left(e^{\alpha_{2}^{*}} z\right)+\ldots+a_{n} f\left(e^{\alpha_{n}^{*}} z\right)=0, z \in \mathbb{C},
$$

where $\alpha_{j}^{*}$ is so that $\log \left(e^{\alpha_{j}^{*}}\right)=\alpha_{j}$ for each $j=1,2, \ldots, n$. That is, $\alpha_{j}^{*}=\operatorname{Re} \alpha_{j}+$ $i\left(\operatorname{Im} \alpha_{j}+2 \pi k_{j}\right)$ for some $k_{j} \in \mathbb{Z}$ such that $\operatorname{Im} \alpha_{j}+2 \pi k_{j} \in[-\pi, \pi)$ for each $j=$ $1,2, \ldots, n$.

Analogously, $Q(z)$ is the corresponding exponential polynomial associated to the functional equation

$$
b_{1} f\left(e^{\beta_{1}^{*}} z\right)+b_{2} f\left(e^{\beta_{2}^{*}} z\right)+\ldots+b_{n} f\left(e^{\beta_{n}^{*}} z\right)=0, z \in \mathbb{C},
$$

where $\beta_{j}^{*}$ is so that $\log \left(e^{\beta_{j}^{*}}\right)=\beta_{j}$ for each $j=1,2, \ldots, n$. Therefore, since $\beta_{j}^{*}=$ $\operatorname{Re} \beta_{j}+i\left(\operatorname{Im} \beta_{j}+2 \pi m_{j}\right)$ for some $m_{j} \in \mathbb{Z}$ and $\beta_{j}=\alpha_{j}+\lambda$ for $j=1,2, \ldots, n$, we have

$$
e^{\beta_{j}^{*}}=e^{\beta_{j}}=e^{\lambda} e^{\alpha_{j}}=e^{\lambda} e^{\alpha_{j}^{*}}
$$

and, consequently, functional equations (2.2) and (2.3) are equivalent in $\mathcal{F}_{n}$ and the result follows. 
We proved above that there exists a clear correspondence between the binary relations defined on the classes $\mathcal{P}$ and $\mathcal{F}_{n}$. In fact, two exponential polynomials with the same set of zeros are closely related to two equivalent functional equations.

Furthermore, the equivalent functional equations in $\mathcal{F}_{n}$ have the same set of solutions defined on whole $\mathbb{C}$, as we nextly prove.

Proposition 2.9 For each integer number $n \geq 2$, two equivalent functional equations in $\mathcal{F}_{n}$ have the same solutions defined on $\mathbb{C}$.

Proof. Let

$$
w_{1} f\left(a_{1} z\right)+w_{2} f\left(a_{2} z\right)+\ldots+w_{n} f\left(a_{n} z\right)=0, z \in \mathbb{C},
$$

and

$$
v_{1} f\left(b_{1} z\right)+v_{2} f\left(b_{2} z\right)+\ldots+v_{n} f\left(b_{n} z\right)=0, z \in \mathbb{C},
$$

be two equivalent functional equations in $\mathcal{F}_{n}$ and $c, \gamma \in \mathbb{C}$, with $c \neq 0$, such that $v_{j}=c w_{j}$ and $b_{j}=a_{j} e^{\gamma}$ for each $j=1,2, \ldots, n$. First, let $f_{1}(z)$ be a solution of (2.4), defined on $\mathbb{C}$, then $f_{1}(z)$ satisfies $(2.5)$ if and only if

$$
c w_{1} f_{1}\left(a_{1} e^{\gamma} z\right)+c w_{2} f_{1}\left(a_{2} e^{\gamma} z\right)+\ldots+c w_{n} f_{1}\left(a_{n} e^{\gamma} z\right)=0,
$$

which, by replacing $z$ by $\frac{z}{e^{\gamma}}$, is equal to

$$
w_{1} f_{1}\left(a_{1} z\right)+w_{2} f_{1}\left(a_{2} z\right)+\ldots+w_{n} f_{1}\left(a_{n} z\right)=0,
$$

that is true.

Conversely, let $f_{2}(z)$ be a solution of $(2.5)$, defined on $\mathbb{C}$, then $f_{2}(z)$ satisfies $(2.4)$ if and only if

$$
\frac{v_{1}}{c} f\left(b_{1} e^{-\gamma} z\right)+\frac{v_{2}}{c} f\left(b_{2} e^{-\gamma} z\right)+\ldots+\frac{v_{n}}{c} f\left(b_{n} e^{-\gamma} z\right)=0,
$$

which, by replacing $z$ by $z e^{\gamma}$, is equal to

$$
v_{1} f_{2}\left(b_{1} z\right)+v_{2} f_{2}\left(b_{2} z\right)+\ldots+v_{n} f_{2}\left(b_{n} z\right)=0,
$$

that is also true.

As we said in the introduction, the importance of this result lies in the fact that the binary relation established on the set of functional equations of the form (1.2), which by Proposition 2.7 leads to exponential polynomials with the same set of zeros, determines not only the same solutions $g_{n, j}(z)$ of the form (2.1) closely related to the exponential polynomials of the class $\mathcal{P}$, but also any other solutions defined on $\mathbb{C}$ of equivalent functional equations.

\section{Real frequencies}

We now particularize the definitions of the preceding section to the case of exponential polynomials with complex coefficients and real frequencies. 
Definition 3.1 For each integer number $n \geq 1$, consider exponential polynomials of the form

$$
a_{1} e^{\alpha_{1} z}+\ldots+a_{n} e^{\alpha_{n} z}, z \in \mathbb{C}
$$

with $\alpha_{1}<\ldots<\alpha_{n}$ and $a_{j} \in \mathbb{C}$ for each $j=1, \ldots, n$. An exponential polynomial is said to be of the class $\mathcal{P}_{0}$ when it is of the form (3.1).

Definition 3.2 An exponential polynomial is said to be of the class $\mathcal{T}_{0} \subset \mathcal{P}_{0}$ if it is of the form

$$
c e^{\lambda z}, c \in \mathbb{C}, \lambda \in \mathbb{R} \text {. }
$$

Note that the sets $\mathcal{P}_{0}$ and $\mathcal{T}_{0}$ of functions of the form (3.1) and (3.2) respectively are also closed with respect to the sum and the multiplication and it is verified $\mathcal{P}_{0} \subset \mathcal{P}$ and $\mathcal{T}_{0} \subset \mathcal{T}$.

By analogy with Definition 2.3, we now identify two elements of the class $\mathcal{P}_{0}$ in terms of the class $\mathcal{T}_{0}$.

Definition 3.3 We will say that two elements $P(z), Q(z) \in \mathcal{P}_{0}$ are equivalent, and it will be denoted by $P(z) \sim_{0} Q(z)$, when there exists $T(z) \in \mathcal{T}_{0}$ such that $P(z)=T(z) \cdot Q(z)$ for all $z \in \mathbb{C}$.

Note that the binary relation $\sim_{0}$ defined above on the class $\mathcal{P}_{0}$ is also an equivalence relation.

An important result is that Proposition 2.4 can be extended to this case. Hence, two exponential polynomials in $\mathcal{P}_{0}$ with the same set of zeros and same multiplicity can be directly identified by the equivalence relation $\sim_{0}$, which is more specific than the relation $\sim$ previously considered.

Theorem 3.4 Let $P(z)$ and $Q(z)$ be two exponential polynomials in the class $\mathcal{P}_{0}$. Thus $P(z) \sim_{0} Q(z)$ if and only if $Z(P)=Z(Q)$ and their zeros have associated the same multiplicity.

Proof. First, let $P(z)$ and $Q(z)$ be two exponential polynomials in the same equivalence class of $\mathcal{P}_{0} / \sim_{0}$. Thus, there exists $T(z)=c e^{\lambda z} \in \mathcal{T}_{0}$ such that $P(z)=T(z) Q(z)$. Without loss of generality, let $z_{0} \in Z(Q)$ be a zero of $Q(z)$ of multiplicity $k \geq 1$, that is,

$$
Q\left(z_{0}\right)=Q^{\prime}\left(z_{0}\right)=Q^{\prime \prime}\left(z_{0}\right)=\ldots=Q^{(k-1)}\left(z_{0}\right)=0, Q^{(k)}\left(z_{0}\right) \neq 0 .
$$

Therefore, $P\left(z_{0}\right)=0$ and, since

$$
P^{(m)}\left(z_{0}\right)=C e^{\lambda z_{0}} \sum_{j=0}^{m}\left(\begin{array}{c}
m \\
j
\end{array}\right) \lambda^{m-j} Q^{(j)}\left(z_{0}\right), 1 \leq m \leq k,
$$

we have

$$
P\left(z_{0}\right)=P^{\prime}\left(z_{0}\right)=P^{\prime \prime}\left(z_{0}\right)=\ldots=P^{(k-1)}\left(z_{0}\right)=0, P^{(k)}\left(z_{0}\right) \neq 0 .
$$

Therefore $z_{0}$ is also a zero of $P(z)$ of multiplicity $k$. 
Conversely, suppose that $P(z)$ and $Q(z)$ are two exponential polynomials of the form (3.1) so that

$$
Z(P)=Z(Q)=\left\{z_{1}, z_{2}, \ldots, z_{l}, \ldots\right\}
$$

and their zeros have associated the same multiplicity. Then, by Hadamard factorization theorem [2, Theorem 4.4.3], we have

$$
P(z)=C_{P} e^{A_{P} z} J(z)
$$

and

$$
Q(z)=C_{Q} e^{A_{Q} z} J(z)
$$

where $C_{P}, C_{Q}, A_{P}, A_{Q} \in \mathbb{C}$ and $J(z)=z^{k} \prod_{l \geq 1} E_{h}\left(\frac{z}{z_{l}}\right)$ with $k$ the order of the zero at $z=0$ and $h$ an integer number depending on the exponent of convergence of $\left\{z_{l}\right\}$, where

$$
E_{h}(z)= \begin{cases}1-z & \text { if } h=0 \\ (1-z) \exp \left(z+\frac{z^{2}}{2}+\ldots+\frac{z^{h}}{h}\right) & \text { if } h \geq 1\end{cases}
$$

Therefore, since $P(z)$ and $Q(z)$ are exponential polynomials, from (3.3) and (3.4), $J(z)$ is also an exponential polynomials of the form

$$
c_{1} e^{\gamma_{1} z}+\ldots+c_{n} e^{\gamma_{n} z}
$$

with $c_{j}, \gamma_{j} \in \mathbb{C}, j=1, \ldots, n$ and $n$ the number of terms of the exponential polynomials $P(z)$ and $Q(z)$. Furthermore, if $P(z)$ and $Q(z)$ are of the form

$$
a_{1} e^{\alpha_{1} z}+\ldots+a_{n} e^{\alpha_{n} z}
$$

and

$$
b_{1} e^{\beta_{1} z}+\ldots+b_{n} e^{\beta_{n} z}
$$

respectively, with $\alpha_{1}<\ldots<\alpha_{n}$ and $\beta_{1}<\ldots<\beta_{n}$, also from (3.3) and (3.4) we obtain

$$
\gamma_{1}=\alpha_{1}-A_{P}, \ldots, \gamma_{n}=\alpha_{n}-A_{P}
$$

and

$$
\gamma_{1}=\beta_{1}-A_{Q}, \ldots, \gamma_{n}=\beta_{n}-A_{Q}
$$

Consequently,

$$
\beta_{k}-\alpha_{k}=\beta_{j}-\alpha_{j}
$$


for all $j, k \in\{1, \ldots, n\}, j \neq k$, or equivalently there exists $\lambda \in \mathbb{R}$ such that $\beta_{j}=\alpha_{j}+\lambda$ for each $j=1, \ldots, n$. Hence,

$$
\lambda=A_{P}-A_{Q} \in \mathbb{R} .
$$

On the other hand, we have

$$
P(z)=\frac{Q(z)}{C_{Q} e^{A_{Q} z}} C_{P} e^{A_{P} z},
$$

i.e.

$$
P(z)=T(z) Q(z)
$$

where $T(z)=c e^{\lambda z}$ with $c=\frac{C_{P}}{C_{Q}} \neq 0$ and $\lambda=A_{P}-A_{Q} \in \mathbb{R}$. So $P(z) \sim_{0} Q(z)$.

This development can be again translated to the functional equations of the form (1.2) with real coefficients $\gamma_{j}$ 's. Thus consider another binary relation on the class of functional equations of the form

$$
a_{1} f\left(\gamma_{1} z\right)+a_{2} f\left(\gamma_{2} z\right)+\ldots+a_{n} f\left(\gamma_{n} z\right)=0, z \in \mathbb{C},
$$

where $n \geq 2$ is an integer number, $a_{j} \in \mathbb{C} \backslash\{0\}$ and $\gamma_{j} \in \mathbb{R} \backslash\{0\}$ for each $j=$ $1,2, \ldots, n$.

Recall that the corresponding exponential polynomial (see Definition 2.5) associated to a functional equation of the form (3.5) is given by

$$
P_{n}(z)=a_{1} e^{z \log \gamma_{1}}+a_{2} e^{z \log \gamma_{2}}+\ldots+a_{n} e^{z \log \gamma_{n}} .
$$

Definition 3.5 For each integer number $n \geq 2$, let $\mathcal{G}_{n, 0}$ be the set of all functional equations of the form (3.5) with $n$ terms. We will say that two functionals equations in $\mathcal{G}_{n, 0}$,

$$
a_{1} f\left(\gamma_{1} z\right)+a_{2} f\left(\gamma_{2} z\right)+\ldots+a_{n} f\left(\gamma_{n} z\right)=0, z \in \mathbb{C}
$$

and

$$
b_{1} f\left(\delta_{1} z\right)+b_{2} f\left(\delta_{2} z\right)+\ldots+b_{n} f\left(\delta_{n} z\right)=0, z \in \mathbb{C},
$$

are equivalent when there exist $\lambda \in \mathbb{R}$ and $c \in \mathbb{C} \backslash\{0\}$ such that $b_{j}=c a_{j}$ and $\delta_{j}=e^{\lambda} \gamma_{j}$ for each $j=1,2, \ldots, n$.

Given $n \geq 2$, observe that $\mathcal{G}_{n, 0} \subset \mathcal{F}_{n}$. The results obtained for the equivalent functional equations in $\mathcal{F}_{n}$ can be extended to the class $\mathcal{G}_{n, 0}$ with the equivalence class above. Particularly, Propositions 2.7 and 2.9 can be analogously proved for this case and the proof of Proposition 2.8 is nextly adapted.

Proposition 3.6 Let $P(z)$ and $Q(z)$ be two exponential polynomials in $\mathcal{P}_{0} \backslash \mathcal{T}_{0}$ such that $P(z) \sim_{0} Q(z)$. Thus $P(z)$ and $Q(z)$ are the corresponding exponential polynomials associated to two equivalent functional equations in $\mathcal{G}_{n, 0}$ for some integer number $n \geq 2$. 
Proof. As $P(z) \sim_{0} Q(z)$, then

$$
P(z)=c e^{\lambda z} Q(z)
$$

with $c \in \mathbb{C} \backslash\{0\}$ and $\lambda \in \mathbb{R}$. Therefore, if $P(z) \in \mathcal{P}_{0}$ is of the form

$$
P(z)=a_{1} e^{\alpha_{1} z}+\ldots+a_{n} e^{\alpha_{n} z}
$$

for some $n \geq 2$, then

$$
Q(z)=b_{1} e^{\beta_{1} z}+\ldots+b_{n} e^{\beta_{n} z}
$$

with $b_{j}=c a_{j}$ and $\beta_{j}=\alpha_{j}+\lambda$ for $j=1,2, \ldots, n$.

Furthermore, $P(z)$ and $Q(z)$ are the corresponding exponential polynomials associated to the functional equations

$$
a_{1} f\left(e^{\alpha_{1}} z\right)+a_{2} f\left(e^{\alpha_{2}} z\right)+\ldots+a_{n} f\left(e^{\alpha_{n}} z\right)=0, z \in \mathbb{C},
$$

and

$$
b_{1} f\left(e^{\beta_{1}} z\right)+b_{2} f\left(e^{\beta_{2}} z\right)+\ldots+b_{n} f\left(e^{\beta_{n}} z\right)=0, z \in \mathbb{C},
$$

respectively.

Therefore, since $e^{\beta_{j}}=e^{\lambda} e^{\alpha_{j}}$ and $b_{j}=c a_{j}$ for each $j=1,2, \ldots, n$, we infer that functional equations (3.6) and (3.7) are equivalent in $\mathcal{G}_{n, 0}$ and the result follows.

\section{Aligned frequencies}

When the frequencies have the same argument, we can generalize the development of the preceding section in order to consider more specific equivalence classes.

For each integer number $n \geq 1$, consider exponential polynomials of the form

$$
a_{1} e^{\alpha_{1} z}+\ldots+a_{n} e^{\alpha_{n} z}, z \in \mathbb{C},
$$

with complex coefficients and distinct frequencies $\alpha_{1}, \ldots, \alpha_{n}$ such that there exists a complex number $\xi$ with $\operatorname{Arg}(\xi)=\theta \in[0, \pi)$ verifying $\alpha_{j}=r_{j} \xi$ for some $r_{j} \in \mathbb{R}$ and for each $j=1, \ldots, n$. This condition is equivalent to state that the frequencies are aligned, that is, $\operatorname{Arg}\left(\alpha_{j}\right)=\operatorname{Arg}\left(\alpha_{k}\right) \pm \pi$ for $j, k \in\{1, \ldots, n\}$ with $j \neq k$.

Definition 4.1 Let $P(z)=a_{1} e^{\alpha_{1} z}+\ldots+a_{n} e^{\alpha_{n} z}$ be an exponential polynomial of the form (4.1) and $\xi \in \mathbb{C}$ with $\operatorname{Arg}(\xi)=\theta \in[0, \pi)$ so that $\alpha_{j}=r_{j} \xi$ for some $r_{j} \in \mathbb{R}$ and for each $j=1, \ldots, n$. Thus, we will say that $P(z)$ is of the class $\mathcal{P}_{\theta}$.

Equivalently, given $i \in\{1, \ldots, n\}$, the number $\theta \in[0, \pi)$ is given by

$$
\theta=\left\{\begin{array}{l}
\operatorname{Arg}\left(\alpha_{i}\right)+\pi \text { if } \operatorname{Arg}\left(\alpha_{i}\right)<0 \\
\operatorname{Arg}\left(\alpha_{i}\right) \quad \text { if } \operatorname{Arg}\left(\alpha_{i}\right) \geq 0
\end{array} .\right.
$$

Definition 4.2 An exponential polynomial is said to be of the class $\mathcal{T}_{\theta} \subset \mathcal{P}_{\theta}$ if it is of the form $c e^{\lambda z}$, with $c, \lambda \in \mathbb{C}$ such that $\operatorname{Arg}(\lambda)=\theta$ or $\operatorname{Arg}(\lambda)=\theta-\pi$. 
Definition 4.3 We will say that two elements $P(z), Q(z) \in \mathcal{P}_{\theta}$ are equivalent, and it will be denoted by $P(z) \sim_{\theta} Q(z)$, when there exists $T(z) \in \mathcal{T}_{\theta}$ such that $P(z)=T(z) \cdot Q(z)$ for all $z \in \mathbb{C}$.

The results obtained in the previous sections can be extended to the class $\mathcal{P}_{\theta}$ with the equivalence relation $\sim_{\theta}$. To do it, given $P(z) \in \mathcal{P}_{\theta}$, we define the function

$$
H_{P}(z):=P\left(z e^{-i \theta}\right) \in \mathcal{P}_{0}
$$

Then, with the usual notation $Z(f)$ for the set of zeros of a function $f$, we have

$$
Z(P)=e^{-i \theta} Z\left(H_{P}\right)
$$

and, consequently, the zeros of $P(z)$ will be obtained multiplying by $e^{-i \theta}$ the zeros of $H_{P}(z)$.

Lemma 4.4 Let $P(z)$ and $Q(z)$ be two exponential polynomials of the class $\mathcal{P}_{\theta}$. Consider $H_{P}(z):=P\left(z e^{-i \theta}\right)$ and $H_{Q}(z):=Q\left(z e^{-i \theta}\right)$. Thus $P(z) \sim_{\theta} Q(z)$ if and only if $H_{P}(z) \sim_{0} H_{Q}(z)$.

Proof. If $P(z) \sim_{\theta} Q(z)$, there exists $T_{1}(z) \in \mathcal{T}_{\theta}$ such that $P(z)=T_{1}(z) \cdot Q(z)$ for all $z \in \mathbb{C}$. Hence $P\left(z e^{-i \theta}\right)=T_{1}\left(z e^{-i \theta}\right) \cdot Q\left(z e^{-i \theta}\right)$ for all $z \in \mathbb{C}$, i.e. $H_{P}(z)=T_{2}(z)$. $H_{Q}(z)$ for all $z \in \mathbb{C}$, with $T_{2}(z):=T_{1}\left(z e^{-i \theta}\right) \in \mathcal{T}_{0}$. Therefore $H_{P}(z) \sim_{0} H_{Q}(z)$.

Conversely, if $H_{P}(z)$ and $H_{Q}(z)$ are equivalent in $\mathcal{P}_{0}$, there exists $T_{3}(z) \in \mathcal{T}_{0}$ such that $H_{P}(z)=T_{3}(z) \cdot H_{Q}(z)$ for all $z \in \mathbb{C}$. Hence $H_{P}\left(z e^{i \theta}\right)=T_{3}\left(z e^{i \theta}\right) \cdot H_{Q}\left(z e^{-i \theta}\right)$ for all $z \in \mathbb{C}$ or, equivalently, $P(z)=T_{4}(z) \cdot Q(z)$ for all $z \in \mathbb{C}$, with $T_{4}(z):=$ $T_{3}\left(z e^{i \theta}\right) \in \mathcal{T}_{\theta}$.

In this manner, we prove the following result.

Corollary 4.5 Let $P(z)$ and $Q(z)$ be two exponential polynomials of the class $\mathcal{P}_{\theta}$. Thus $P(z) \sim_{\theta} Q(z)$ if and only if $Z(P)=Z(Q)$ and their zeros have associated the same multiplicity.

Proof. The demonstration is obtained just by considering $H_{P}(z):=P\left(z e^{-i \theta}\right) \in \mathcal{P}_{0}$ and $H_{Q}(z):=Q\left(z e^{-i \theta}\right) \in \mathcal{P}_{0}$, and by using (4.2), Lemma 4.4 and Theorem 3.4.

Finally, we can consider another binary relation on the class of functional equations of the form

$$
a_{1} f\left(\gamma_{1} z\right)+a_{2} f\left(\gamma_{2} z\right)+\ldots+a_{n} f\left(\gamma_{n} z\right)=0, z \in \mathbb{C}
$$

where $n \geq 2$ is an integer number, $a_{j} \in \mathbb{C} \backslash\{0\}$ and the $\gamma_{j}$ 's are $\theta$-aligned, that is $\operatorname{Arg}\left(\gamma_{j}\right)=\operatorname{Arg}\left(\gamma_{k}\right) \pm \pi$ for $j, k \in\{1, \ldots, n\}, j \neq k$, and $\theta \in[0, \pi)$ defined as

$$
\theta:=\left\{\begin{array}{l}
\operatorname{Arg}\left(\gamma_{i}\right)+\pi \text { if } \operatorname{Arg}\left(\gamma_{i}\right)<0 \\
\operatorname{Arg}\left(\gamma_{i}\right) \quad \text { if } \operatorname{Arg}\left(\gamma_{i}\right) \geq 0
\end{array}\right.
$$

Recall that the corresponding exponential polynomial associated to a functional equation of the form (4.3) is given by

$$
P_{n}(z)=a_{1} e^{z \log \gamma_{1}}+a_{2} e^{z \log \gamma_{2}}+\ldots+a_{n} e^{z \log \gamma_{n}} .
$$


Definition 4.6 For each integer number $n \geq 2$, let $\mathcal{G}_{n, \theta}$ be the set of all functional equations of the form (4.3) with $n$ terms. We will say that two functionals equations in $\mathcal{G}_{n, \theta}$,

$$
a_{1} f\left(\gamma_{1} z\right)+a_{2} f\left(\gamma_{2} z\right)+\ldots+a_{n} f\left(\gamma_{n} z\right)=0, z \in \mathbb{C}
$$

and

$$
b_{1} f\left(\delta_{1} z\right)+b_{2} f\left(\delta_{2} z\right)+\ldots+b_{n} f\left(\delta_{n} z\right)=0, z \in \mathbb{C}
$$

are equivalent when there exist $\lambda \in \mathbb{C}$ such that $\operatorname{Arg}(\lambda)=\theta$ or $\operatorname{Arg}(\lambda)=\theta-\pi$, and $c \in \mathbb{C} \backslash\{0\}$ such that $b_{j}=c a_{j}$ and $\delta_{j}=e^{\lambda} \gamma_{j}$ for each $j=1,2, \ldots, n$.

The next proposition is an immediate corollary of Lemma 4.4 and Proposition 3.6. In fact, the demonstration of the second part of it is obtained just by following verbatim the proof of Proposition 3.6.

Proposition 4.7 Let $P(z)$ and $Q(z)$ be two exponential polynomials in $\mathcal{P}_{\theta} \backslash \mathcal{T}_{\theta}$ such that $P(z) \sim_{\theta} Q(z)$. Thus

i) $H_{P}(z):=P\left(z e^{-i \theta}\right)$ and $H_{Q}(z):=Q\left(z e^{-i \theta}\right)$ are the corresponding exponential polynomials associated to two equivalent functional equations in $\mathcal{G}_{n, 0}$ for some integer number $n \geq 2$;

ii) $P(z)$ and $Q(z)$ are the corresponding exponential polynomials associated to two equivalent functional equations in $\mathcal{G}_{n, \theta}$ for some integer number $n \geq 2$.

For the case of exponential polynomials of real coefficients we can make an analogous development in order to consider more specific equivalence classes which preserve the preceding properties. For example, consider the following definitions.

Definition 4.8 For each integer number $n \geq 1$, consider exponential polynomials of the form

$$
a_{1} e^{\alpha_{1} z}+\ldots+a_{n} e^{\alpha_{n} z}, z \in \mathbb{C}
$$

with $\theta$-aligned frequencies and $a_{j} \in \mathbb{R}$ for each $j=1, \ldots, n$. Thus, an exponential polynomial is said to be of the class $\mathcal{P}_{\theta, r}$ when it is of the form (4.4).

Observe that the class $\mathcal{P}_{0, r}$ is formed by exponential polynomials of real coefficients and frequencies, which constitutes an important case studied in the literature through for example the partial sums of the Riemann zeta function $1+2^{-z}+\ldots+n^{-z}$, where $n$ is an integer number greater than or equal to 2 .

Definition 4.9 An exponential polynomial is said to be of the class $\mathcal{T}_{\theta, r} \subset \mathcal{P}_{\theta, r}$ if it is of the form $c e^{\lambda z}$, with $c \in \mathbb{R}$ and $\lambda \in \mathbb{C}$ such that $\operatorname{Arg}(\lambda)=\theta$ or $\operatorname{Arg}(\lambda)=\theta-\pi$.

Note that the sets $\mathcal{P}_{\theta, r}$ and $\mathcal{T}_{\theta, r}$ are again closed with respect to the sum and the multiplication and it is verified $\mathcal{P}_{\theta, r} \subset \mathcal{P}_{\theta} \subset \mathcal{P}$ and $\mathcal{T}_{\theta, r} \subset \mathcal{T}_{\theta} \subset \mathcal{T}$. Just as in the other cases, we will identify two elements in the class $\mathcal{P}_{\theta, r}$ in terms of the class $\mathcal{T}_{\theta, r}$

Definition 4.10 We will say that two elements $P(z), Q(z) \in \mathcal{P}_{\theta, r}$ are equivalent, and it will be denoted by $P(z) \sim_{\theta, r} Q(z)$, when there exists $T(z) \in \mathcal{T}_{\theta, r}$ such that $P(z)=T(z) \cdot Q(z)$ for all $z \in \mathbb{C}$.

The results previously obtained can be easily extended to the class $\mathcal{P}_{\theta, r}$ with the equivalence relation $\sim_{\theta, r}$. 
Example 4.11 Let $P(z):=1+e^{z \log 2}+e^{z \log 3}, Q(z):=e^{z i}+e^{z(\log 2+i)}+e^{z(\log 3+i)}$ and $R(z):=e^{z \log 2}+e^{z \log 4}+e^{z \log 6}$. Observe that $P(z), Q(z), R(z) \in \mathcal{P}$, $P(z), R(z) \in \mathcal{P}_{0, r} \subset \mathcal{P}_{0} \subset \mathcal{P}$ and we have the following equalities:

$$
Q(z)=e^{i z} P(z), R(z)=e^{z \log 2} P(z), R(z)=e^{z(i-\log 2)} Q(z)
$$

Therefore $P(z) \sim Q(z), P(z) \sim R(z)$ and $Q(z) \sim R(z)$ and, consequently, $Z(P)=$ $Z(Q)=Z(R)$. Furthermore, it is also verified $P(z) \sim_{0} R(z)$ and $P(z) \sim_{0, r} R(z)$, which is a more specific relation because the number of transformations in the class $\mathcal{T}_{0, r}$ is smaller than that of $\mathcal{T}_{0}$ and much smaller than that of $\mathcal{T}$.

\section{References}

[1] J.M. Almira and Kh. F. Abu-Helaiel, On solutions of $f(x)+f\left(a_{1} x\right)+\ldots+$ $f\left(a_{N} x\right)=0$ and related equations, Ann. Tiberiu Popoviciu Semin. Funct. Equ. Approx. Convexity. 9 (2011), 3-17.

[2] R. B. Ash, Complex Variables, Academic Press, London, 1971.

[3] G. Mora, Y. Cherruault and A. Ziadi, Functional equations generating spacedensifying curves, Computers and Math. with Appl. 39 (2000), 45-55.

[4] D. G. Dickson, Asymptotic Distribution of Exponential Sums, Publ. Math. Debrecen. 11 (1964), 295-300.

[5] G. MorA, A note on the functional equation $F(z)+F(2 z)+\cdots+F(n z)=0$, J. Math. Anal. Appl. 340 (2008), 466-475.

[6] G. Mora And J.M. SePulcre, The zeros of Riemann zeta partial sums yield solutions to $f(x)+f(2 x)+\ldots+f(n x)=0$, Mediterr. J. Math. 10 (2013), 3, 1221-1232.

[7] G. PolyA, Geometiisches ber die Verteilung der Nullstellen gewisser ganzer trans zendenterFunktionen, Munchener Sitzungsberichte. 50 (1920), 285-290.

[8] A. J. VAn der Poorten and R. Tijdeman, On common zeros of exponential polynomials, Enseignament Math. 21 (1975), 1, 57-67.

[9] C.E. Wilder, Expansion problems of ordinary linear differential equations with auxiliary conditions at more than two points, Trans. Am. Math. Soc. 18 (1917), 415-442. 